\title{
Pulse Excitation Scheme for the Analysis of Wide-Band Characteristics of Optical Waveguides
}

\author{
Junji Yamauchi, Member, IEEE, Shin Sasaki, and Hisamatsu Nakano, Fellow, IEEE
}

\begin{abstract}
A new pulse excitation scheme using a Fourier series is proposed to analyze the wide-band response of optical waveguides. To validate the scheme, a waveguide with high-reflection coatings and a waveguide grating are analyzed using the finite-difference time-domain method. Calculation shows that the transmissivity and reflectivity can be evaluated by a one-time solution over a wide wavelength range of $0.5-2.0 \mu \mathrm{m}$ (two octaves).
\end{abstract}

Index Terms-Finite-difference time-domain (FDTD) methods, high-reflection (HR) coatings, optical waveguides, pulse analysis, waveguide grating.

\section{INTRODUCTION}

I $\mathrm{N}$ THE time-domain lightwave analysis, such as the finitedifference time-domain (FDTD) method [1], pulse excitation with the aid of the Fourier transform is often used, since this allows us to obtain the wide-band response of the lightwave by a one-time solution.

It should be noted that an optical waveguide supports substantially different transverse field distributions, depending on a specific wavelength. Nevertheless, the conventional Gaussian-pulse method assumes the same input modeshape for all wavelengths. This often results in serious error at wavelengths away from the carrier wavelength of the pulse, particularly in strongly guiding waveguides. We, therefore, often need accuracy checking based on continuous-wave (CW) inputs [2]. One way to avoid this error is to use so-called "bootstrapping" [1], which requires running a preliminary model of the waveguide that is sufficiently long to decay all undesired fields. However, this requires tedious work with an extra time-consuming procedure.

In this letter, we propose a new pulse excitation scheme using a superposed wave composed of the modal fields at several wavelengths. As an application, a waveguide with high-reflection (HR) coatings [3] and a waveguide grating [4] are studied to demonstrate the effectiveness of the present excitation scheme. Only the numerical results for the transverse-electric mode are presented, although the transverse-magnetic mode can also be treated. The present scheme enables us to obtain the wide-band response of the transmissivity and reflectivity by a one-time solution.

Manuscript received June 28, 2004; revised August 12, 2004

The authors are with the Faculty of Engineering, Hosei University, Koganei, Tokyo 184-8584, Japan (e-mail: j.yma@k.hosei.ac.jp)

Digital Object Identifier 10.1109/LPT.2004.839020

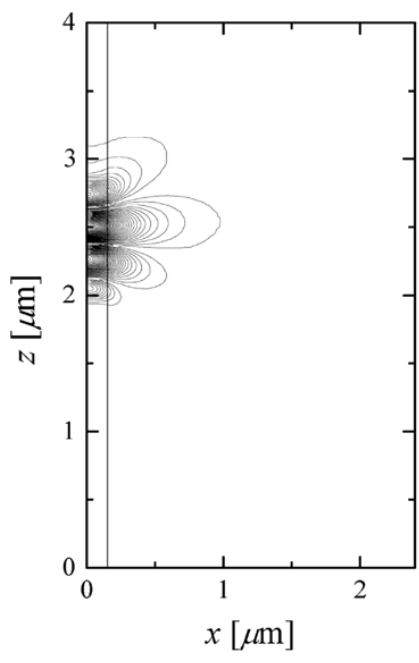

(a)

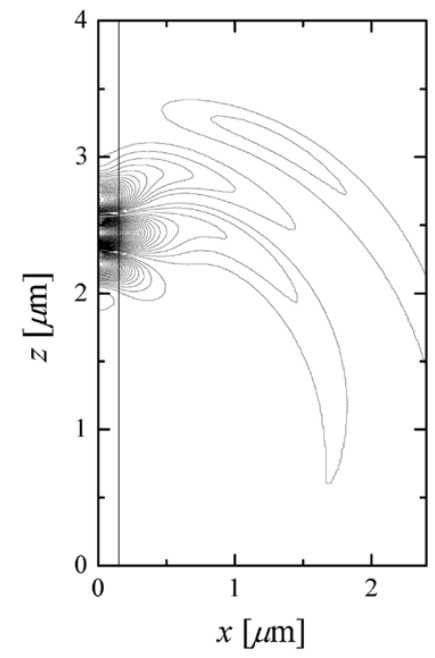

(b)
Fig. 1. Instantaneous snapshots of pulses propagating in a slab waveguide, in which the refractive indexes are $n_{\mathrm{co}}=1.5$ and $n_{\mathrm{cl}}=1.0$, and the core width is $w=0.3 \mu \mathrm{m}$. The fields are excited in the $+z$ direction at $z=1.0 \mu \mathrm{m}$, and observed at $t=10 \mathrm{fs}$. Half the region is illustrated. The maximum field is normalized to unity, and the field contour is plotted in steps of 0.02. (a) Present pulse and (b) conventional Gaussian pulse.

\section{EXCITATION SCHEME}

In the evaluation of wavelength characteristics using a time-domain method, a Gaussian pulse modulated by a sine wave is often used [5], which is given by

$$
E_{y, \text { inc }}(x, t)=E_{y}(x) \exp \left[-\left(\frac{t-t_{0}}{\tau}\right)^{2}\right] \sin \omega_{c}\left(t-t_{0}\right)
$$

where $t_{0}$ is the time offset, $\tau$ is the pulsewidth parameter, $\omega_{c}$ is $2 \pi c / \lambda_{c}$, in which $\lambda_{c}$ is the carrier wavelength, and $E_{y}(x)$ is the eigenmode field of the waveguide at $\lambda_{c}$. It is needless to say that the eigenmode field varies as the wavelength deviates from the carrier wavelength. The conventional Gaussian-pulse method cannot take into account the difference in modal profile at wavelengths far away from the carrier wavelength, resulting in undesirable radiation as the field propagates [as will be seen in Fig. 1(b)].

In order to resolve the problem, we introduce the Fourier series

$$
E_{y, \text { inc }}(x, t)=\sum_{n=1}^{\infty} E_{y}^{n}(x) b_{n} \sin n \omega_{0} t, \quad\left(0 \leq t \leq 2 t_{0}\right)
$$




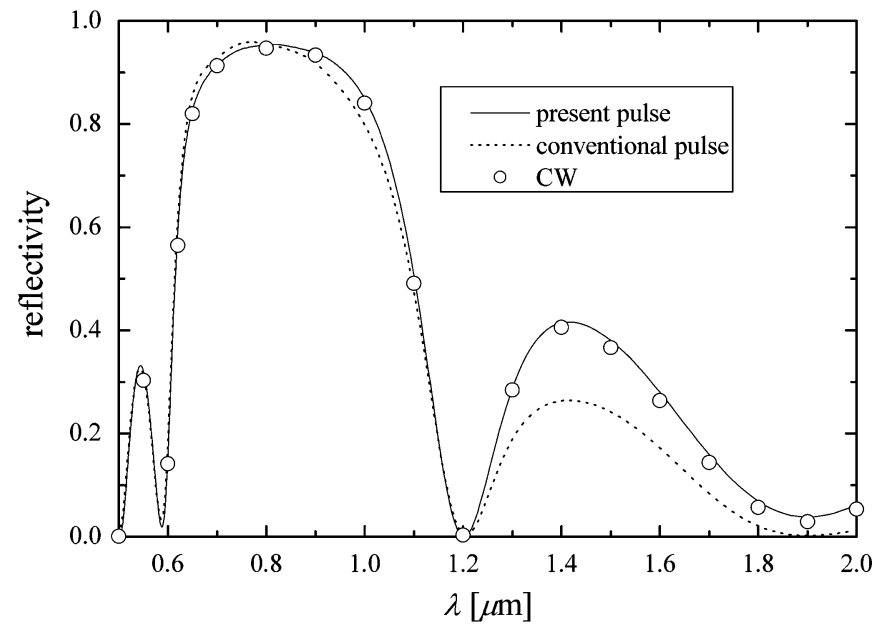

Fig. 2. Reflectivity versus wavelength. The carrier wavelength is chosen to be $\lambda_{c}=0.8 \mu \mathrm{m}$.

where $\omega_{0}\left(=2 \pi c / \lambda_{0}=2 \pi / T_{0}\right)$ is the fundamental angular frequency, in which $\lambda_{0}$ is the fundamental wavelength, and the coefficient of the $n$th Fourier component is given by

$$
\begin{aligned}
b_{n}=\frac{2}{T_{0}} \int_{0}^{T_{0}}\left\{\exp \left[-\left(\frac{t-t_{0}}{\tau}\right)^{2}\right]\right. \\
\\
\left.\quad \times \sin \omega_{c}\left(t-t_{0}\right) \sin n \omega_{0} t\right\} d t
\end{aligned}
$$

in which $t_{0}$ is taken to be $T_{0} / 2$. We should note in (2) that $E_{y}^{n}(x)$ can comprise the modal transverse fields at angular frequencies of $\omega_{0}, 2 \omega_{0}, 3 \omega_{0}, \ldots$. As expected, the accuracy improves as the number of Fourier components is increased. However, three components are enough to obtain reasonable results, as will be seen later. Note that we have some freedom to choose $\omega_{c}$ and $\omega_{0}$. In this letter, we choose $\omega_{0}$ in such a way that its higher components include $\omega_{c}$.

To clarify the essential properties of the present pulse, we first analyze the pulse propagation in a two-dimensional (2-D) waveguide. We let $\tau=t_{0} / 2$, the carrier wavelength $\lambda_{c}=0.8 \mu \mathrm{m}$, and the fundamental wavelength $\lambda_{0}=1.6 \mu \mathrm{m}$. Then the coefficients $b_{n}$ are calculated to be $b_{1} \cong-0.24, b_{2} \cong 0.44, b_{3} \cong-0.24, b_{4} \cong 0.04, \ldots$. in which $b_{n}$ higher than $b_{3}$ may be neglected because of their smallness. Thus, the three wavelengths to be used are $1.6 \mu \mathrm{m}$, $1.6 / 2=0.8 \mu \mathrm{m}$, and $1.6 / 3 \cong 0.53 \mu \mathrm{m}$, which are effective beyond a wide wavelength range of $0.53-1.6 \mu \mathrm{m}$, as will be shown in Fig. 2.

The instantaneous snapshot of the present pulse is shown in Fig. 1(a). For comparison, the field obtained with the conventional Gaussian pulse in (1) is also illustrated in Fig. 1(b). It is worth mentioning that the unguided field observed in Fig. 1(b) is almost eliminated in Fig. 1(a).

\section{APPLICATION}

To demonstrate the effectiveness of the present scheme, we deal with two 2-D structures. The spectral responses of the transmissivity and reflectivity are evaluated from the ratio between the Fourier transforms of the observed pulse and the excited

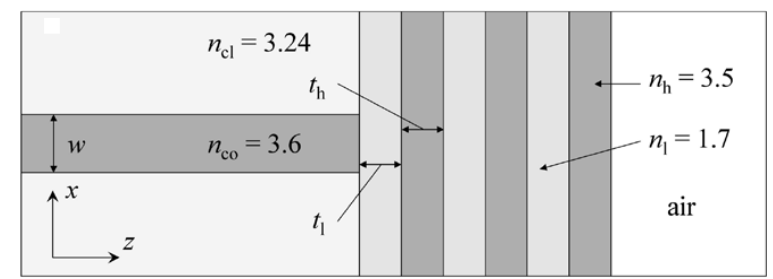

Fig. 3. Configuration of a waveguide with HR coatings. There are three periods.

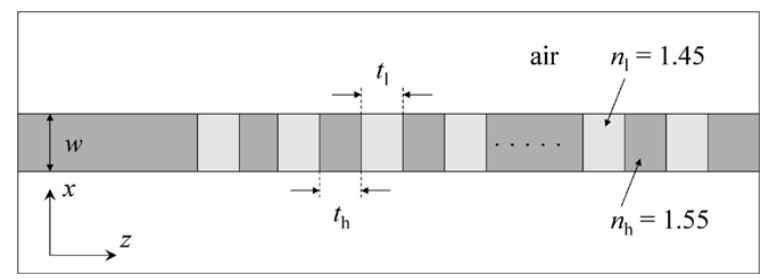

Fig. 4. Configuration of a waveguide grating. There are 16 grating periods.

pulse. For comparison, the results obtained with the conventional Gaussian pulse and with the $\mathrm{CW}$ inputs are also presented. Throughout this letter, the perfectly matched layer absorbing boundary condition [6] is imposed at the edge of the computational window.

\section{A. Waveguide With HR Coatings}

We investigate the reflectivity of the waveguide with HR coatings shown in Fig. 3, which is the same as that treated in [3]. The HR coatings are composed of six alternating dielectric layers of a quarter-wavelength (at $\lambda=0.8 \mu \mathrm{m}$ ) thickness. The refractive indexes are $n_{\mathrm{co}}=3.6, n_{\mathrm{cl}}=3.24, n_{h}=3.5$, and $n_{l}=1.7$. The core width is $w=0.3 \mu \mathrm{m}$. The numerical parameters are fixed to be $\Delta x=w / 24=0.0125 \mu \mathrm{m}$ and $\Delta z=0.004 \mu \mathrm{m}$.

We use the same pulse parameters as those used in Fig. 1. Fig. 2 shows the reflectivity as a function of wavelength. It is found that the reflectivity obtained with the present scheme is in good agreement with that obtained with the $\mathrm{CW}$ inputs. The results obtained with the conventional Gaussian pulse deviate particularly at long wavelengths, since the carrier wavelength is fixed to be $0.8 \mu \mathrm{m}$.

\section{B. Waveguide Grating}

We next consider the waveguide grating shown in Fig. 4, which is the same as that treated in [4]. The refractive indexes are $n_{h}=1.55$ and $n_{l}=1.45$. The grating thicknesses are taken to be $t_{h}=0.3276 \mu \mathrm{m}$ and $t_{l}=0.3103 \mu \mathrm{m}$ to meet the phase-matching condition. There are 16 periods. The core width is fixed to be $w=0.3 \mu \mathrm{m}$. The numerical parameters are chosen to be $\Delta x=w / 20=0.015 \mu \mathrm{m}$ and $\Delta z=\left(t_{h}+t_{l}\right) / 37 \cong$ $0.017 \mu \mathrm{m}$.

We use the following pulse parameters: $\tau=t_{0} / 2, \lambda_{c}=$ $1.5 \mu \mathrm{m}$, and $\lambda_{0}=4.5 \mu \mathrm{m}$. This choice leads to coefficients of $b_{1} \cong-0.04, b_{2} \cong 0.24, b_{3} \cong-0.44, b_{4} \cong$ $0.24, b_{5} \cong-0.04, \ldots$. We neglect $b_{1}$ and $b_{n}$ higher than $b_{4}$, so that the wavelength components of the Fourier series are $4.5 / 2=2.25 \mu \mathrm{m}, 4.5 / 3=1.5 \mu \mathrm{m}$, and $4.5 / 4=1.125 \mu \mathrm{m}$. This set of wavelengths is appropriate for the analysis over a range of longer wavelengths as compared with that used in 


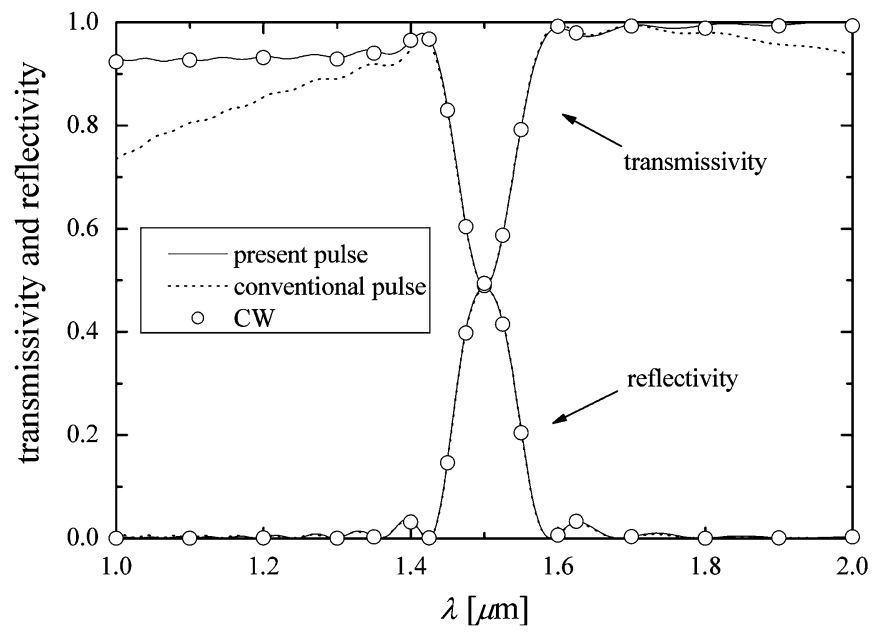

Fig. 5. Transmissivity and reflectivity versus wavelength. The carrier wavelength is chosen to be $\lambda_{c}=1.5 \mu \mathrm{m}$.

Fig. 2. Fig. 5 shows the transmissivity and reflectivity against wavelength. We can again find good agreement between the present method and the $\mathrm{CW}$ input method. The transmissivity obtained with the conventional Gaussian pulse induces error as the wavelength deviates from a carrier wavelength of $1.5 \mu \mathrm{m}$.

\section{CONCLUSION}

A pulse excitation scheme using a superposed wave composed of the modal fields at several wavelengths has been pro- posed for the analysis of wide-band characteristics of optical waveguides. The present pulse can propagate along an optical waveguide without undergoing radiation.

To validate the present scheme, a waveguide with HR coatings and a waveguide grating have been analyzed using the FDTD method. The result obtained with the present scheme agrees well with that obtained with the $\mathrm{CW}$ inputs, while the result obtained with the conventional Gaussian pulse induces error at wavelengths away from the carrier wavelength. The present scheme contributes to reduction in computational time, since the transmissivity and reflectivity over a wide range of wavelengths are obtained by only a one-time solution.

\section{REFERENCES}

[1] A. Taflove and S. C. Hagness, Computational Electrodynamics: The Finite-Difference Time-Domain Method. Norwood, MA: Artech House, 2000.

[2] R. L. Espinola, R. U. Ahmad, F. Pizzuto, M. J. Steel, and R. M. Osgood Jr., "A study of high-index-contrast $90^{\circ}$ waveguide bend structures," Opt. Express, vol. 8, no. 9, pp. 517-528, 2001.

[3] J. Yamauchi, H. Kanbara, and H. Nakano, "Analysis of optical waveguides with high-reflection coatings using the FD-TD method," IEEE Photon. Technol. Lett., vol. 10, no. 1, pp. 111-113, Jan. 1998.

[4] W. P. Huang, S. P. Chu, A. Goss, and S. K. Chaudhuri, "A scalar finitedifference time-domain approach to guided-wave optics," IEEE Photon. Technol. Lett., vol. 3, no. 6, pp. 524-526, Jun. 1991.

[5] S. T. Chu, W. P. Huang, and S. K. Chaudhuri, "Simulation and analysis of waveguide based optical integrated circuits," Comp. Phys. Commun., vol. 68, pp. 451-484, 1991.

[6] J. P. Berenger, "A perfectly matched layer for the absorption of electromagnetic waves," J. Comp. Phy., vol. 114, no. 2, pp. 185-200, 1994. 